\title{
Front Matter: Volume 6770
}

, "Front Matter: Volume 6770," Proc. SPIE 6770, Fiber Optic Sensors and Applications V, 677001 (29 October 2007); doi: 10.1117/12.7744830

SPIE. Event: Optics East, 2007, Boston, MA, United States 


\section{PROCEEDINGS OF SPIE}

\section{Fiber Optic Sensors and Applications $V$}

Eric Udd

Editor

10-12 September 2007

Boston, Massachusetts, USA

Sponsored and Published by

SPIE

Volume 6770 
The papers included in this volume were part of the technical conference cited on the cover and title page. Papers were selected and subject to review by the editors and conference program committee. Some conference presentations may not be available for publication. The papers published in these proceedings reflect the work and thoughts of the authors and are published herein as submitted. The publisher is not responsible for the validity of the information or for any outcomes resulting from reliance thereon.

Please use the following format to cite material from this book:

Author(s), "Title of Paper," in Fiber Optic Sensors and Applications V, edited by Eric Udd, Proceedings of SPIE Vol. 6770 (SPIE, Bellingham, WA, 2007) Article CID Number.

ISSN 0277-786X

ISBN 9780819469304

Published by

SPIE

P.O. Box 10, Bellingham, Washington 98227-0010 USA

Telephone +1 3606763290 (Pacific Time) · Fax +1 3606471445

SPIE.org

Copyright (C 2007, Society of Photo-Optical Instrumentation Engineers

Copying of material in this book for internal or personal use, or for the internal or personal use of specific clients, beyond the fair use provisions granted by the U.S. Copyright Law is authorized by SPIE subject to payment of copying fees. The Transactional Reporting Service base fee for this volume is $\$ 18.00$ per article (or portion thereof), which should be paid directly to the Copyright Clearance Center (CCC), 222 Rosewood Drive, Danvers, MA 01923. Payment may also be made electronically through CCC Online at copyright.com. Other copying for republication, resale, advertising or promotion, or any form of systematic or multiple reproduction of any material in this book is prohibited except with permission in writing from the publisher. The CCC fee code is $0277-786 \times / 07 / \$ 18.00$.

Printed in the United States of America.

Publication of record for individual papers is online in the SPIE Digital Library.

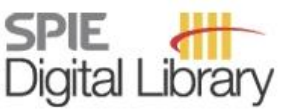

SPIEDigitallibrary.org

Paper Numbering: Proceedings of SPIE follow an e-First publication model, with papers published first online and then in print and on CD-ROM. Papers are published as they are submitted and meet publication criteria. A unique, consistent, permanent citation identifier (CID) number is assigned to each article at the time of the first publication. Utilization of CIDs allows articles to be fully citable as soon they are published online, and connects the same identifier to all online, print, and electronic versions of the publication. SPIE uses a six-digit CID article numbering system in which:

- The first four digits correspond to the SPIE volume number.

- The last two digits indicate publication order within the volume using a Base 36 numbering system employing both numerals and letters. These two-number sets start with 00, 01, 02, 03, 04, 05, $06,07,08,09,0 A, 0 B \ldots 0 Z$, followed by $10-12,20-2 Z$, etc.

The CID number appears on each page of the manuscript. The complete citation is used on the first page, and an abbreviated version on subsequent pages. Numbers in the index correspond to the last two digits of the six-digit CID number. 


\section{Contents}

vii Conference Committee

\section{SESSION 1 FIBER GRATING SENSORS I}

677002 Review of multi-parameter fiber grating sensors (Invited Paper) [6770-01]

E. Udd, Columbia Gorge Research, LLC (USA)

677003 Optical fiber distributed sensing structural health monitoring (SHM) strain measurements taken during cryotank $Y$-joint test article load cycling at liquid helium temperatures [6770-02]

S. G. Allison, W. H. Prosser, D. A. Hare, T. C. Moore, W. S. Kenner, NASA Langley Research Ctr. (USA)

677004 Fiber Bragg grating based measurement of elastic properties at cryogenic temperatures [6770-03]

I. Latka, W. Ecke, B. Höfer, T. Habisreuther, Institute of Photonic Technology (Germany);

J. P. Kallmeyer, Max Planck Institute for Plasma Physics (Germany)

$677005 \quad$ Field test investigation of fiber optic seismic geophone in oilfield exploration [6770-04] Y. Zhang, J. Ning, Stevens Institute of Technology (USA); S. Yang, Stevens Institute of Technology (USA) and Yantai Univ. (China); H.-L. Cui, Stevens Institute of Technology (USA)

\section{SESSION 2 FIBER GRATING SENSORS II}

677006 An FBG microphone array system with signal demodulator using AWG [6770-05]

K. Nakamura, T. Fujisue, S. Ueha, Tokyo Institute of Technology (Japan)

677007 Multiplexed strain sensing by synthesis of optical coherence function with time-division phase shift modulation [6770-06]

K. Hotate, K. Matsuo, K.-Y. Song, Z. He, Univ. of Tokyo (Japan)

SESSION $3 \quad$ FIBER GRATING SENSORS III

677009 Femtosecond laser inscribed high temperature fiber Bragg grating sensors (Invited Paper) [6770-08]

S. J. Mihailov, D. Grobnic, R. B. Walker, H. Ding, F. Bilodeau, C. W. Smelser, Communications Research Ctr. Canada (Canada)

6770 OB Design of high sensitivity refractometer based on temperature independent TE and TM modes in open top ridge waveguides [6770-10]

X. Dai, S. J. Mihailov, R. B. Walker, Communications Research Ctr. (Canada); C. Chen, J. Albert, Carleton Univ. (Canada) 
6770 OC Optical fiber static strain sensors: reliability issues [6770-11]

I. Saxena, K. Hui, Intelligent Optical Systems, Inc. (USA)

6770 OD Development of an FBG-based low temperature measurement system for cargo containment of LNG tankers (Invited Paper) [6770-12]

D. G. Kim, W. Yoo, Simulation Tech Inc. (South Korea); P. Swinehart, Lake Shore Cryotronics Inc. (USA); B. Jiang, T. Haber, Micron Optics Inc. (USA); A. Mendez, MCH Engineering, LLC (USA)

\section{SESSION 4 DISTRIBUTED FIBER SENSORS I}

6770 OF Swept-wavelength interferometric interrogation of fiber Rayleigh scatter for distributed sensing applications (Invited Paper) [6770-14]

D. K. Gifford, S. T. Kreger, A. K. Sang, M. E. Froggatt, R. G. Duncan, M. S. Wolfe, B. J. Soller, Luna Technologies (USA)

6770 OG Multiplexed fiber optic acoustic sensors in a $120 \mathrm{~km}$ loop using RF modulation [6770-15] I. C. M. Littler, J. H. Chow, D. A. Shaddock, D. E. McClelland, M. B. Gray, Australian National Univ. (Australia)

$6770 \mathrm{OH} \quad$ Autonomous measurement system for localization of loss-induced perturbation based on transmission-reflection analysis [6770-16]

V. V. Spirin, S. V. Miridonov, E. Mitrani, C. Morales, M. G. Shlyagin, M. Castro, M. Manriquez, Ctr. de Investigación Científica y de Educación Superior de Ensenada (Mexico)

\section{SESSION 5 DISTRIBUTED FIBER SENSORS II}

6770 0J Distributed fiber strain sensor with 1 kHz sampling rate based on Brillouin optical correlation domain analysis [6770-18]

K.-Y. Song, K. Hotate, Univ. of Tokyo (Japan)

6770 OK Intruder signature analysis from a phase-sensitive distributed fiber-optic perimeter sensor [6770-19]

C. K. Madsen, T. Bae, T. Snider, Texas A\&M Univ. (USA)

\section{SESSION 6 OPTICAL FIBERS FOR SENSORS}

6770 0M Specialty fibers for fiber optic sensor application (Invited Paper) [6770-21]

K. Bennett, J. Koh, J. Coon, C. K. Chien, A. Artuso, X. Chen, D. Nolan, M.-J. Li, Corning Inc. (USA)

6770 OP A POF-based distributed strain sensor with intrinsic memory effect [6770-24]

T. Fukumoto, K. Nakamura, S. Ueha, Tokyo Institute of Technology (Japan)

\section{SESSION 7 FIBER OPTIC SENSOR APPLICATIONS AND DEVICES I}

$67700 Q \quad$ Geophysical applications of optical fiber sensors (Invited Paper) [6770-25]

M. A. Zumberge, Univ. of California, San Diego (USA) 
6770 OS Packaging optical sensors for the real world (Invited Paper) [6770-27]

W. Kachmar, K. C. Nardone, ADC Optical Research and Development (USA)

\section{SESSION 8 FIBER OPTIC SENSOR APPLICATIONS AND DEVICES II}

6770 OT Sensor applications of photosensitive capillary waveguides (Invited Paper) [6770-28]

D. J. Markos, K. H. Smith, T. E. Dimmick, L. Dickens, J. P. Barber, W. E. Edwards, Harris Corp. (USA)

6770 OU Ultra-miniature all-glass Fabry-Pérot pressure sensor manufactured at the tip of a multimode optical fiber [6770-29]

É. Pinet, FISO Technologies Inc. (Canada); E. Cibula, D. Đonlagić, Univ. of Maribor (Slovenia)

$67700 \mathrm{~V}$ Minimally invasive fiber probes for optical in-vivo oxygen measurements with integrated optical thermometer [6770-30]

H.-G. Löhmannsröben, E. Schmälzlin, Univ. of Potsdam (Germany)

6770 OW High frequency Pound-Drever-Hall sensing of ring resonator cavities [6770-31]

J. P. Chambers, C. K. Madsen, H. Lin, Texas A\&M Univ. (USA)

$67700 X$ The effect of delay line on the performance of a fiber optic interferometric sensor [6770-32] Y.-L. Lin, K.-H. Lin, National Sun Yat-Sen Univ. (Taiwan); W.-W. Lin, Ta Jen Univ. (Taiwan); M.-H. Chen, National Sun Yat-Sen Univ. (Taiwan)

6770 OY Wireless fiber optic sensor system for strain and pressure measurements on a rotor blade [6770-33]

Y. Liu, A. Lacher, Univ. of Maryland, College Park (USA); G. Wang, A. Purekar,

Techno-Sciences, Inc. (USA); M. YU, Univ. of Maryland, College Park (USA)

$67700 Z$ A debris flow monitoring system by means of fiber optic interferometers [6770-34]

Y.-L. Lin, K.-H. Lin, National Sun Yat-Sen Univ. (Taiwan); W.-W. Lin, Ta Jen Univ. (Taiwan); M.-H. Chen, National Sun Yat-Sen Univ. (Taiwan)

SESSION 9 FIBER OPTIC SENSOR APPLICATIONS AND DEVICES III

677010 Increased throughput single molecule detection of DNA [6770-35]

R. Gurjar, M. Seetamraju, N. Kolodziejski, R. Myers, C. Staples, J. Christian, M. R. Squillante,

G. Entine, Radiation Monitoring Devices, Inc. (USA)

677011 Modulation in optical fiber lasers using polarization feedback [6770-36]

J. Hernández-Cordero, N. Cuando-Espitia, Univ. Nacional Autónoma de México (Mexico)

POSTER SESSION

677014 Experimental investigation of Sagnac beat signals using semiconductor fiber-optic ring laser gyroscope (S-FOG) based on semiconductor optical amplifier (SOA) [6770-43]

S. Tamura, K. Inagaki, H. Noto, T. Harayama, ATR Wave Engineering Labs. (Japan) 
677015 Combined distributed temperature sensing and current monitoring [6770-44]

S. G. M. Krämer, Technische Univ. München (Germany) and GE Global Research

(Germany); W. Feichter, GE Global Research (Germany); F. Puente León, Technische Univ.

München (Germany); J. Stromberger, GE Global Research (Germany)

677017 Optical current sensor using phase shift algorithms [6770-46]

J. L. Flores, G. García-Torales, V. H. Ortiz, Univ. of Guadalajara (Mexico)

677018 A review on optical current transducers for power system metering [6770-47]

V. H. Ortiz, J. L. Flores, G. García-Torales, Univ. of Guadalajara (Mexico)

677019 Long period grating-based fiber-optic PH sensor for ocean monitoring [6770-49]

K. Wang, D. Klimov, Z. Kolber, Monterey Bay Aquarium Research Institute (USA)

6770 1B Analysis of optical-power redistribution for hybrid optical fibers [6770-51]

J. Skapa, V. Vašinek, P. Šiška, VSB-TU Technical Univ. of Ostrava (Czech Republic)

6770 1C Trace gas detection using a novel cantilever-based photoacoustic spectrometer with multiplexed optical fiber-coupled diode lasers and fiber-amplification [6770-52]

A. M. Parkes, K. A. Keen, E. D. McNaghten, AWE plc (United Kingdom)

Author Index 


\title{
Conference Committee
}

\author{
Symposium Chairs
}

Tuan Vo-Dinh, Duke University (USA)

Robert A. Lieberman, Intelligent Optical Systems, Inc. (USA)

Conference Chair

Eric Udd, Columbia Gorge Research, LLC (USA)

Program Committee

Richard O. Claus, Virginia Polytechnic Institute and State University (USA)

Brian Culshaw, University of Strathclyde (United Kingdom)

John P. Dakin, University of Southampton (United Kingdom)

Anthony D. Dandridge, Naval Research Laboratory (USA)

Wolfgang Ecke, Institut für Physikalische Hochtechnologie e.V. (Germany)

Tom W. Graver, Micron Optics, Inc. (USA)

Eric D. Haugse, The Boeing Company (USA)

Kazuo Hotate, University of Tokyo (Japan)

Stephen T. Kreger, Luna Technologies Inc. (USA)

Paul Lefebvre, LXSiX Photonics Inc. (Canada)

Robert A. Lieberman, Intelligent Optical Systems, Inc. (USA)

Michael A. Marcus, Eastman Kodak Company (USA)

Alexis Méndez, MCH Engineering LLC (USA)

Anna G. Mignani, Istituto di Fisica Applicata Nello Carrara (Italy)

Glen A. Sanders, Honeywell Technology (USA)

\section{Session Chairs}

1 Fiber Grating Sensors I

Eric Udd, Columbia Gorge Research, LLC (USA)

Alexis Méndez, MCH Engineering LLC (USA)

2 Fiber Grating Sensors II

Eric Udd, Columbia Gorge Research, LLC (USA)

Stephen J. Mihailov, Communications Research Center Canada (Canada) 
3 Fiber Grating Sensors III

Stephen J. Mihailov, Communications Research Center Canada (Canada)

Kazuo Hotate, University of Tokyo (Japan)

4 Distributed Fiber Sensors I

Steven T. Kreger, Luna Innovations Inc. (USA)

5 Distributed Fiber Sensors II

Robert J. Schroeder, Schlumberger (USA)

6 Optical Fibers for Sensors

Eric Udd, Columbia Gorge Research, LLC (USA)

7 Fiber Optic Sensor Applications and Devices I

Alexis Méndez, MCH Engineering LLC (USA)

8 Fiber Optic Sensor Applications and Devices II

Paul Lefebvre, LxSiX Photonics Inc. (Canada)

$9 \quad$ Fiber Optic Sensor Applications and Devices III

Paul Lefebvre, LxSiX Photonics Inc. (Canada) 